\title{
KIC 9406652: A laboratory for tilted disks in cataclysmic variable stars. II. Modeling of the orbital light curves
}

\author{
Mariko KIMURA ${ }^{1, *}$ and Yoji OSAKI ${ }^{2}$ \\ ${ }^{1}$ Institute of Physical and Chemical Research (RIKEN), 2-1 Hirosawa, Wako, Saitama \\ 351-0198 \\ ${ }^{2}$ Department of Astronomy, School of Science, University of Tokyo, Hongo, Tokyo 113-0033 \\ *E-mail: mariko.kimura@riken.jp \\ Received; Accepted
}

\begin{abstract}
KIC 9406652, one of the recently identified IW And-type dwarf novae, is the best target for studying the tilted disk in cataclysmic variable stars. In a previous paper by Kimura, Osaki, and Kato (2020), we analyzed its Kepler light curves and found that its orbital light curves during the brightening stage were dominated by the reflection effect of the secondary star and varied with the orientation of the tilted disk; the amplitude was maximized at the minimum of the superorbital signal and the phase of the light maximum shifted to an earlier one with the advance of the super-orbital phase. We argued there that this was the direct evidence of the retrogradely precessing tilted disk as the secondary star acts like a reflecting object. In order to confirm this interpretation, we have performed numerical modeling of orbital light curves in this paper. We have succeeded in reproducing the main characteristics of the observed orbital light curves by a simple model in which the secondary star is irradiated by the tilted disk. We have also constrained the inclination angle, $i$, of the binary system and the tilt angle, $\theta$, of the disk purely from photometric considerations. The best-fitting parameter set is found to be $i \sim 45 \mathrm{deg}$ and $\theta \sim 2.0$ deg, respectively. The orbital inclination thus estimated is consistent with that obtained from the spectroscopic considerations within the uncertainty limit. On the other hand, the tilt angle of the disk could be underestimated by using only the semi-amplitude of super-orbital signals.
\end{abstract}

Key words: accretion, accretion disks - novae, cataclysmic variables - stars: dwarf novae - stars: individual (KIC 9406652)

\section{Introduction}

In the previous paper (Kimura et al. 2020b; hereafter referred to as Paper I), we have extensively studied the Kepler light curve of a cataclysmic variable star, KIC 9406652. There we have demonstrated that this star is one of the best laboratories for tilted disks in cataclysmic variables (CVs) as it offers the first direct evidence that the accretion disk is in fact tilted out of the binary orbital plane and retrogradely precessing from analyses of light curves of three periodic signals: orbital, negative superhump, and super-orbital signals. The star, KIC 9406652, is a CV sub-classified as IW And stars, or anomalous Z Cam stars. IW And stars are a small group of dwarf novae (DNe) which exhibit a characteristic light curve: a repetition of a quasistandstill (i.e., a mid-brightness interval with (damping) oscillations) terminated by small-amplitude brightening, which is called "IW And-type phenomenon" by Kato (2019). The typical repetition cycle is around 50 days. More detailed information on IW And stars is given in the introduction of Paper I. 
The present paper is an extension of Paper I, in which we try to model the orbital light curves in this star based on the tilted disk.

The signature of the tilted disk manifests itself in the light curve most clearly by the two periodic light variations, i.e., negative superhumps and super-orbital signals. Negative superhumps are small-amplitude periodic light modulations having a period slightly shorter than the orbital period, and superorbital signals are wavy light modulations with a period much longer than the orbital period (typically around 20 times of the orbital one). In the research on CVs, these two periodic signals were discovered for the first time in TV Col (Motch 1981; Bonnet-Bidaud et al. 1985) and Bonnet-Bidaud et al. (1985) interpreted that these two periodic signals originate from a retrogradely precessing tilted accretion disk, inspired by the observations of some X-ray binaries (e.g., Katz 1973; Leibowitz 1984). Negative superhumps and/or super-orbital signals in various types of CVs were detected in the subsequent observational studies (e.g., Harvey et al. 1995; Patterson et al. 1997; Armstrong et al. 2013), and the source of these periodicities has been investigated. Currently, negative superhumps are thought to be produced by variable brightness of the bright spot sweeping on the tilted disk surface (Wood et al. 2000; Murray et al. 2002; Wood \& Burke 2007). In line with this interpretation, super-orbital signals are thought to be produced by varying projection area of the tilted disk to the observer as its nodal line slowly precesses (Patterson 1999; Patterson et al. 2002). Besides, various mechanisms for the origin of disk tilt have been proposed, but no consensus has been reached. For instance, Lubow (1992) suggested that the 3:1 resonance may amplify perturbations of a disk in the vertical direction. Smak (2009) advocated a model in that variable vertical velocity component of the gas stream, which arises from variable irradiation of the secondary star by the primary star due to the shadow of a tilted, precessing disk, maintains disk tilt. Montgomery \& Martin (2010) proposed that the disk is forced to be tilted by a kind of buoyancy called "lift" and this idea was tested by SPH simulations (Montgomery 2012).

KIC 9406652, is an ideal star to study the tilted disk because it exhibits both negative superhumps and super-orbital light modulations and because the comprehensive Kepler light curve extending over 4 years is available (Gies et al. 2013). We analyzed its Kepler light curve in Paper I and one of the most important findings concerns the orbital light curves in the brightening stage of the IW And-type phenomenon. It has turned out that the orbital light curve is dominated by the effect of irradiation of the secondary star in the brightening stage (see the top panel of Fig. 8 of Paper I) and the orbital light curve varied greatly with the super-orbital phase, i.e., with the orientation of the tilted disk to the observer. The amplitude of the orbital light curve was found to be maximized at the minimum of the super-orbital signals, i.e., when the orientation of the tilted disk is directed opposite to the observer, and the maximum of the orbital light curve was found to be shifted to earlier orbital phases as the super-orbital phase advances (see, Fig. 14 of Paper I). We argued in Paper I that the above-mentioned fact offers direct evidence that the accretion disk is tilted out of the binary orbital plane and its nodal line rotates retrogradely as the secondary star acts like a reflecting mirror for the tilted disk.

In Paper I, we did not subtract negative superhump signals from the original light curve in constructing the orbital light curves. However, since the subtraction of negative superhump signals affects the orbital light curves greatly, we first perform this in Sec 2. In Sec. 3, we describe the basic model and the numerical code that we use for the light curve modeling. In Sec. 4, we set the parameters used for modeling. Sec. 5 presents the results of modeling. Sec. 6 is the summary and discussion.

\section{Subtraction of negative superhump signals from orbital light curves}

In this study, we try to model the orbital light curves during the brightening stage based on a model of the tilted disk and the irradiated secondary star. To do so, we must first mention that some revisions are needed to the top panel of Fig. 8 and Fig. 14 of Paper I. In constructing the orbital light curves in these figures, we did not subtract the effect of negative superhumps. Since the beat period between the orbital period and the negative superhump period is exactly equal to the super-orbital period, the orbital light curves with the super-orbital phase are strongly affected by contamination of negative superhumps and it is essential to subtract this effect.

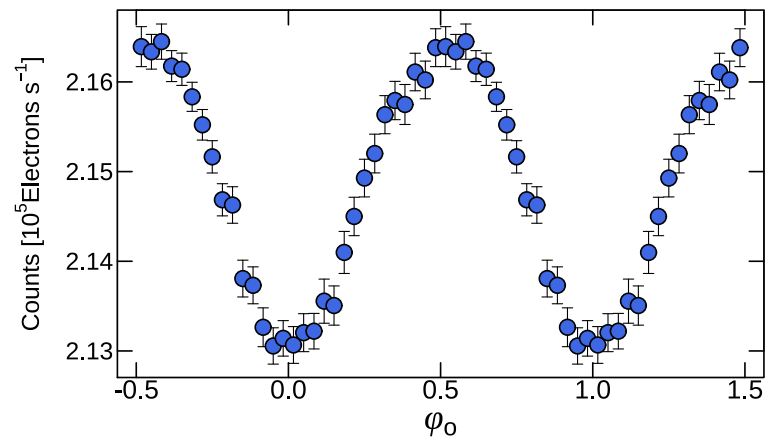

Fig. 1. Phase-averaged profile of orbital signals during brightening in KIC 9406652, which has been constructed after subtracting negative superhump signals from the original data set.

We here subtract negative superhump signals from the original light curve, and reconstruct the orbital light curves. Since the period and light curve profile of negative superhumps were time-varying and the profile sometimes depended on the luminosity of KIC 9406652 (see Fig. 1, Fig. 2, and the left panel of 

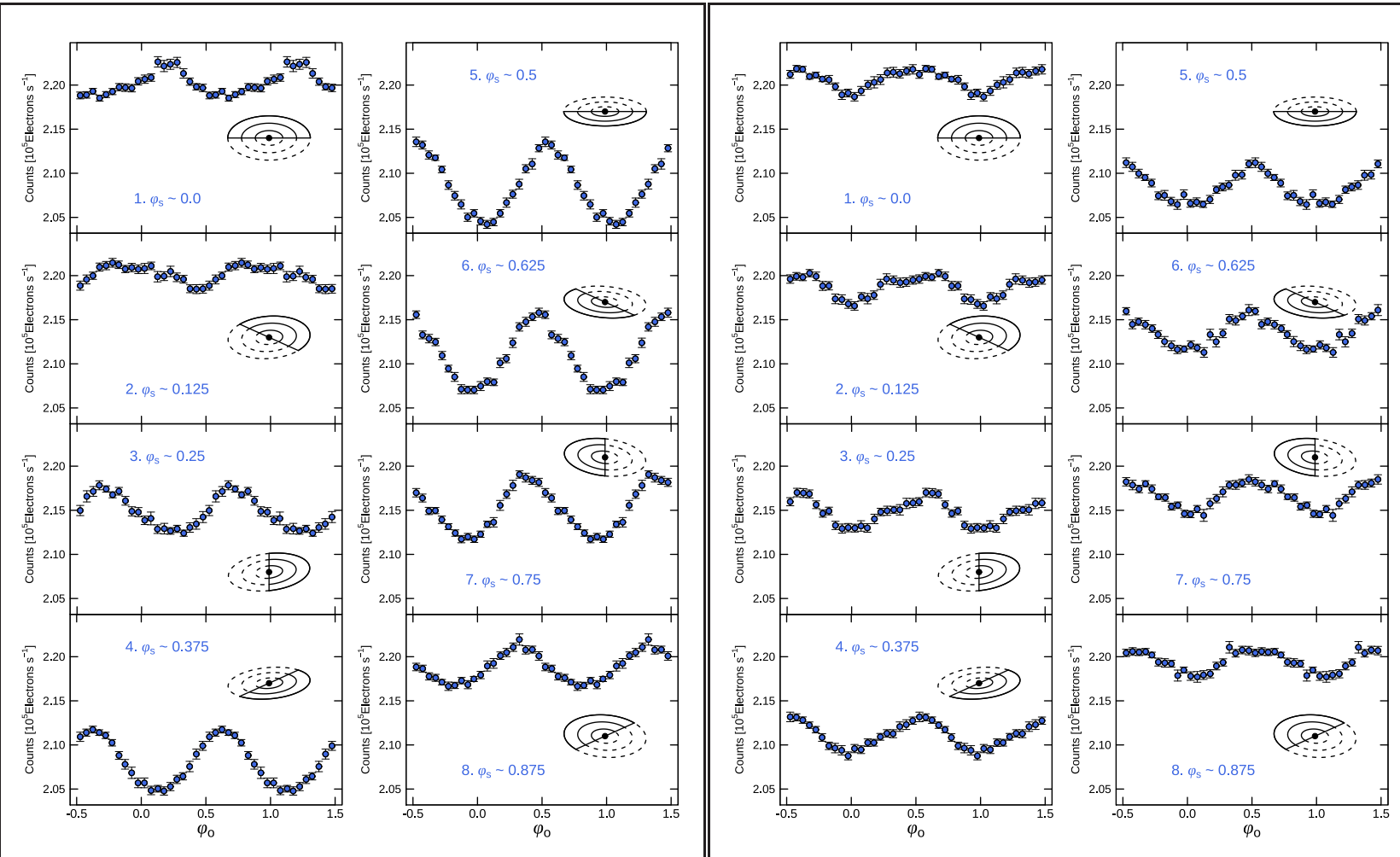

Fig. 2. Comparison of orbital light curves before and after the subtraction of negative superhump signals for eight types of orbital profiles from the data limited for brightening in KIC 9406652. The left figure is the original version same as that in Fig. 14 of Paper I and the right figure is the revised version after the subtraction of negative superhumps. The inset of each figure shows the orientation of the tilted disk where the central WD is marked by the black dot and the diametric line passing the WD is the nodal line of the tilted disk. The solid and dashed lines represent the equatorial plane of the tilted disk above and below the orbital plane of the binary system, respectively. In the right figure, the mean flux level of each light curve is calculated by using the orange circles in Figure 6.

Fig. 3 in Paper I), we divide the observational data into shorter intervals in which we may regard that they are more or less constant. We then construct an average light curve profile of negative superhumps for each interval and subtract it from the original light curve. We finally construct the phase-averaged orbital light curves from the processed light curve in the brightening stage with the same manner as described in subsections 3.3 and 3.5 of Paper I.

Figure 1 and the right panel of Figure 2 are the revised versions to the top panel of Fig. 8 and Fig. 14 of Paper I. For comparison, we also show the original one (Fig. 14 of Paper I) in the left panel of Figure 2. Figure 1 shows the orbital light curve for all data during the brightening stage where $\varphi_{\mathrm{o}}$ is the orbital phase and its phase zero is defined by the inferior conjunction of the secondary star. Figure 2 presents eight orbital light curves for eight different super-orbital phases $\varphi_{\mathrm{s}}$ during the brightening phase. Here the phase zero is defined at the maximum light of super-orbital light modulations and it corresponds to the phase when the front side of the tilted disk turns toward the observer.

Figure 1 is almost the same as the original one of Fig. 8 of Paper I, which means that the orbital profile averaged for all super-orbital phases does not suffer very much from negative superhumps. On the other hand, the orbital light curves for specific super-orbital phases are modified very much by the subtraction of negative superhumps (compare the left and right panels of Figure 2). After the subtraction of negative superhumps, we see that (1) the difference in the amplitude becomes smaller between the orbital light curve at $\varphi_{\mathrm{s}} \sim 0.0$ and that at $\varphi_{\mathrm{s}} \sim 0.5$, (2) the phase shift of the light maximum of orbital light curves with the advance of super-orbital phases becomes less conspicuous, and (3) the light minima of orbital light curves always occur at $\varphi_{\mathrm{o}}=0.0$ while it was not the case in the original ones. However, the main characteristics remain the same before and after this correction as the peak phase shifts to earlier orbital phases as the super-orbital phase advances and the amplitude of the orbital light curve is maximized at $\varphi_{\mathrm{s}}=0.5$.

\section{Basic model and numerical code for modeling light curves}

As discussed in Paper I, the light sources of the orbital signal consist of three components: (1) the orbital hump due to the bright spot produced by the collision of the gas stream with the 
disk at its rim, peaking at the orbital phase $\varphi_{\mathrm{o}}=0.8-0.9,(2)$ the irradiation of the secondary by the tilted accretion disk, peaking around $\varphi_{\mathrm{o}}=0.5$, and (3) the ellipsoidal variation of the secondary star. As well known in $\mathrm{CV}$ s, the relative importance of the three components varies with the system brightness. When the system is faint, i.e., in the quiescence of a dwarf nova, the orbital hump usually dominates in the orbital light curve. When the system brightens in the dwarf-nova outburst, the amplitude of the orbital hump in the orbital light curve decreases with the increase of the system brightness and the orbital hump almost disappears at the outburst maximum. This is because the orbital hump is swamped by the increased background radiation, i.e., the increased disk radiation. As discussed in Paper I and seen in Fig. 8 of Paper I, the same is true in the case of KIC 9406652 and the irradiation of the secondary dominates in the orbital light curve during the brightening stage. In our modeling, we do not consider the gas stream which produces the bright spot at the disk rim (i.e., the orbital hump) during the brightening stage.

The basic model we use here is the standard semi-detached binary model of the cataclysmic variable star, consisting of the WD primary star, the Roche-lobe filling secondary star, and the accretion disk, which contribute to the orbital light curve during the brightening stage. The accretion disk is tilted out of the binary orbital plane with a fixed tilt angle, $\theta$, and its nodal line rotates retrogradely. We do not consider any warping of the disk plane and it is assumed to be coplanar. The accretion disk is assumed to be circular and axisymmetric and it has a fixed outer radius. The secondary star is assumed to be irradiated by the bright tilted disk. The observer looks at this system with the inclination angle, $i$. Figure 3 illustrates our basic model.

The numerical code that we use is the revised version of the code developed by Hachisu \& Kato (2001) who simulated light curves of recurrent novae. Although the method for calculating the flux is the same as that described in Hachisu \& Kato (2001), we apply the response functions provided by Mann \& von Braun (2015) and the Kepler team ${ }^{1}$ in the revised code. In this numerical code, the surfaces of the accretion disk and the secondary star are divided into patches as displayed in Figure 3 , and each patch emits photons by black body radiation at the local temperature. The numbers of surface patch elements are $16 \times 64$ (latitude $\times$ longitude) for each of the northern and the southern hemispheres of the secondary, $16 \times 32$ (radial $\times$ azimuthal) for the tilted disk as for each of the front face (seen from the observer) and the back face. We divide the surface patches for the sidewall of the disk by $2 \times 32$ (upper and lower sides $\times$ azimuthal). The original code by Hachisu \& Kato (2001) can treat irradiation of these two components (i.e., the accretion disk and the secondary) by each other. In our study, we consider only the irradiation of the secondary by the accre-

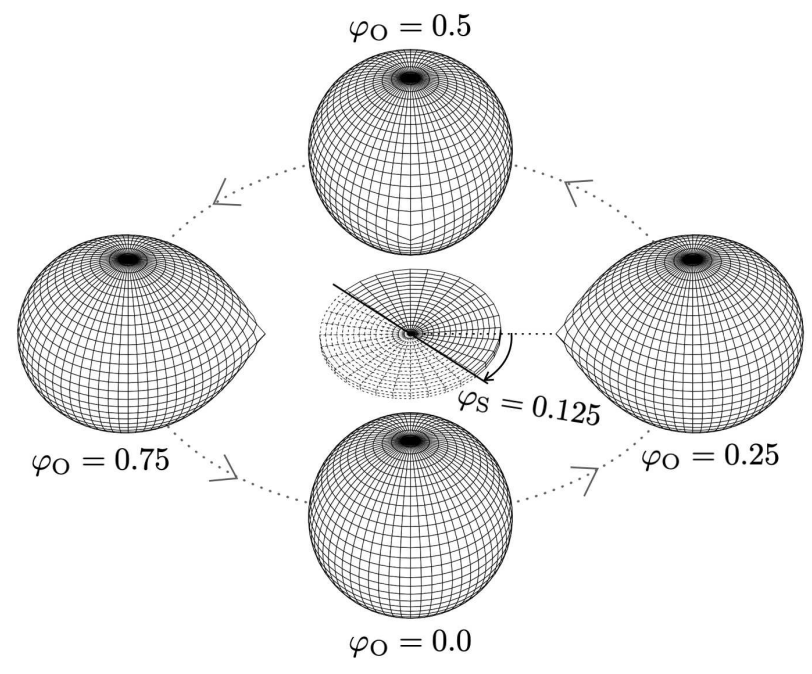

Fig. 3. Schematic figure of our basic model geometry of KIC 9406652 Here, the inclination angle is assumed to be $50 \mathrm{deg}$. The central black point represents a WD and we put a tilted disk at the center with a tilt angle of 4.5 deg for visibility. The nodal line is indicated by a diametric line passing the central WD. The secondary star orbits counterclockwise around the central WD and the tilted disk precesses clockwise. We plot parts of the disk by the solid/dashed lines if its equatorial plane is above/below the orbital plane. The phases, $\varphi_{\mathrm{S}}$ and $\varphi_{\mathrm{o}}$, stand for that of the precession of the tilted disk (or that of the super-orbital modulation) and the orbital phase, respectively. The phase zero for $\varphi_{\mathrm{S}}$ is defined when the nodal line of the tilted disk is perpen dicular to the line of sight and the front face of the tilted disk turns towards the observers. The super-orbital phase in this figure is 0.125 . The orbital phase zero is defined when the secondary star is at the inferior conjunction.

tion disk because the accretion disk is much brighter than the secondary star in the brightening stage of KIC 9406652. The code also includes the emission from the WD, though it is negligible in our case. The numbers of surface patch elements for the WD is $16 \times 32$ (latitude $\times$ longitude). The details of this numerical code are described in Hachisu \& Kato (2001).

As for each surface element on the secondary star, the radiation from the accretion disk visible from the particular surface element is summed up and is used to heat the surface element. Here we introduce an efficiency parameter of the irradiation, $\eta$, in our code, in such a way that a fraction, $\eta$, of the irradiating energy is used to raise the local temperature of the particular surface element. That is, $\eta=1.0$ if all energy received at each surface element by the irradiating radiation is used to raise the temperature of the surface element, and $\eta=0$ if no heating occurs by the irradiation. In our modeling, $\eta$ is assumed to be a free adjustable parameter but it is assumed to take a given value once the inclination is specified.

\section{Parameters for modeling}

\subsection{Binary parameters}

We first reconsider the binary parameters of KIC 9406652 ac${ }^{1}<$ https://nexsci.caltech.edu/workshop/2012/keplergo/CalibrationResponse.shtmleording to Gies et al. (2013) and Kimura et al. (2020b). We set 
the orbital period $\left(P_{\text {orb }}\right)$ to be $0.2545094 \mathrm{~d}$, a value derived in Kimura et al. (2020b). Gies et al. (2013) provided the radial velocity curves of the WD and the secondary star in this object. We have fitted them with sinusoidal curves with the fixed orbital period and have obtained the mass ratio $\left(q \equiv M_{2} / M_{1}\right)$ to be $0.87 \pm 0.08$. Here $M_{1}$ and $M_{2}$ are the masses of the primary and the secondary stars. We thus set the mass ratio to be 0.87 in this study. If the orbital period is well determined, the mass of the Roche-lobe filling secondary star is constrained by the empirical relation investigated by Knigge (2006) as discussed by Gies et al. (2013). We set the secondary mass to be $0.73 M_{\odot}$ from this consideration and therefore the WD mass to be $0.84 M_{\odot}$. The inclination angle is constrained by the following mass function, that is,

$\frac{P_{\text {orb }}}{2 \pi G}=\frac{M_{2} \sin i^{3}}{q(1+q)^{2} K_{2}^{3}}$,

where $G$ is the gravitational constant and $K_{2}$ is the semiamplitude of the radial velocity curve of the secondary star. The orbital inclination is estimated to be $\sim 50$ deg from equation (1). Its possible range determined from the spectroscopic observations is then $45-60 \mathrm{deg}$, if we take into account possible uncertainties in $M_{2}, K$, and $q$. The binary separation ( $a$ ) is calculated from $4 \pi^{2} a^{3}=P_{\mathrm{orb}}^{2} G\left(M_{1}+M_{2}\right)$ and it is estimated to be $1.37 \times 10^{11} \mathrm{~cm}$.

We next set the effective temperature of the WD and the secondary star. The mass accretion rate onto the WD in KIC 9406652 is thought to be high in the brightening stage. The WD temperature $\left(T_{1}\right)$ would become as high as $\sim 40,000 \mathrm{~K}$ in the dwarf-nova outburst (see Table 2.8 in Warner (1995) and Godon et al. (2017)). We, therefore, set $T_{1}$ to be $40,000 \mathrm{~K}$, though its exact value is not important since the emission from the WD is negligible in the optical wavelength range of our interest as described in section 3 . As for the effective temperature of the secondary star $\left(T_{2}\right)$, we first considered a possibility to determine it from the infrared measurements referred in Gies et al. (2013). However, it is difficult to do this because the disk is bright even at infrared wavelengths. On the other hand, according to the empirical relation between the spectral type of the secondary star and the orbital period investigated in Knigge (2006), $T_{2}$ would be estimated to be around $4,370 \mathrm{~K}$ from the orbital period of KIC 9406652 and its dispersion is a few hundred Kelvin. That is, $T_{2} \sim 4,100-4,600 \mathrm{~K}$ is allowed. As will be discussed in the next subsection, the temperature of the secondary star, $T_{2}$ should rather be determined from the flux ratio of the secondary star to the disk and its determination is postponed to the next subsection.

\subsection{Parameters of the accretion disk}

We consider a tilted disk with a tilt angle $\theta$. Its temperature distribution is assumed to be that of the standard disk expressed as

$$
T=T_{*}\left(\frac{r}{r_{\mathrm{in}}}\right)^{-3 / 4}\left[1-\sqrt{\frac{r_{\mathrm{in}}}{r}}\right]^{1 / 4}, T_{*}=\left(\frac{3 G M_{1} \dot{M}}{8 \pi \sigma r_{\mathrm{in}}^{3}}\right)^{1 / 4}
$$

Here, $r, r_{\text {in }}, G, M_{1}, \dot{M}$ and $\sigma$ represent the distance from the central object to a given ring of the disk, the innermost radius of the disk, the gravitational constant, the WD mass, the mass accretion rate at a given radius, and the Stefan-Boltzmann constant, respectively (Shakura \& Sunyaev 1973). We also set the innermost radius of the disk and the WD radius to be $6.0 \times 10^{8} \mathrm{~cm}$ because the WD mass is about $0.84 M_{\odot}$. We assume that the outermost radius of the accretion disk is given by the tidal truncation radius because we study the orbital light curves during the brightening stage. The tidal truncation radius is calculated to be 0.328 in units of the binary separation from the results of Paczynski (1977) for the binary system with the mass ratio $q=0.87$.

Let us now discuss the mass transfer rate and the temperature of the secondary star in our modeling. IW And stars are supposed to be the very cataclysmic binary systems near the borderline between dwarf novae and nova-like variables and thus their mass transfer rate is supposed to be near the critical mass transfer rate $\left(\dot{M}_{\text {crit }}\right)$ above which the disk is thermally stable. In the case of the steady standard disk, the mass-transfer rate is equal to $\dot{M}$ in equation (2). Therefore $\dot{M}$ is estimated to be $10^{17.66} \mathrm{~g} \mathrm{~s}^{-1}$ for the binary parameter of KIC 9406652 (see, Hameury et al. 1998).

In the case of KIC 9406652, the distance is accurately measured to be 348 pc by the Gaia parallax (Bailer-Jones et al. 2018). The $V$-band photometry is performed by the All-Sky Automated Survey for Supernovae (ASAS-SN) data (Davis et al. 2015). On the other hand, the flux ratio, $F_{2} / F_{1}$, for that of the secondary to that of the disk, was derived to be 0.06(2) through the Kepler response function in Paper I based on the result in Gies et al. (2013). It is noted here that this value is derived from optical spectra taken in the quasi-standstill state. We now explore the best set of $\dot{M}$ and $T_{2}$ to satisfy the following two conditions: (1) the $V$-band magnitude is around 11.5-11.9 on average and (2) $F_{2} / F_{1}=0.06(2)$ in quasi-standstills. We have run the numerical code for this purpose and have found that $\dot{M}=10^{17.7} \mathrm{~g} \mathrm{~s}^{-1}$ and $T_{2}=4,100 \mathrm{~K}$ satisfy these conditions over $\eta=0.0-1.0$ and $i=30-55 \mathrm{deg}$ for the distance of KIC 9406652 . The mass transfer rate thus obtained is found to be very near the critical mass transfer rate mentioned above. Since the $V$ band flux is brighter by $\sim 0.3 \mathrm{mag}$ in the brightening phase than that in quasi-standstills, $\dot{M}$ will be $10^{17.9}-10^{18.0} \mathrm{~g} \mathrm{~s}^{-1}$ in the brightening stage. We therefore set $\dot{M}$ to be $10^{18.0} \mathrm{~g} \mathrm{~s}^{-1}$ and $T_{2}=4,100 \mathrm{~K}$ in our modeling during the brightening stage.

We postulate that the thickness of the disk basically follows that of the standard disk. The equation that we used is represented as 
$\frac{H}{r}=1.72 \times 10^{-2} \alpha^{-1 / 10} \dot{M}_{16}^{3 / 20}\left(\frac{M_{1}}{M_{\odot}}\right)^{-3 / 8} r_{10}^{1 / 8}\left[1-\sqrt{\frac{r_{\text {in }}}{r}}\right]^{3 / 5}$

where $H, \dot{M}_{16}$ and $r_{10}$ represent the height of the disk, $\dot{M} / 10^{16} \mathrm{~g} \mathrm{~s}^{-1}$ and $r / 10^{10} \mathrm{~cm}$, respectively (Shakura \& Sunyaev 1973). The $\alpha$ value is assumed to be 0.2 . The thickness of the disk with our binary parameters is then given by $H / r \sim 0.05$ at the outer edge. On the other hand, we may need to integrate the vertical disk structure instead of the one-zone approximation to accurately know the thickness of the disk and Smak (1992) showed the disk in the outburst state has $H / r \sim 0.1$ at the disk outer edge. Since the standard disk is thinner than this, we also study the case of a thicker disk with $H / r=0.1$ at the disk outer edge by multiplying a constant factor to equation (3).

\section{Modeling of the orbital light curve during the brightening stage}

\subsection{Our approach to the problem}

We try to simulate the orbital light curves during the brightening stage of KIC 9406652, i.e., Figure 1 and the right figure of Figure 2 on the basis of our model of the tilted disk and the irradiated secondary star. The purpose is to examine how well our model can reproduce the variation of the observed orbital light curves with respect to the super-orbital phase. The parameters used in our modeling are, (1) the irradiation efficiency, $\eta$, (2) the orbital inclination, $i$, and (3) the tilt angle, $\theta$. As discussed in subsection 4.1, the orbital inclination of KIC 9406652 is constrained to be $45-60$ deg from the spectroscopic considerations. However, we study light curves for a much wider range in the orbital inclination with 30-60 deg and try to see if any preference in the orbital inclination exists from the photometric considerations. As for the tilt angle, $\theta$, we have concluded in Paper I that it was lower than $3 \mathrm{deg}$ from the semi-amplitude of superorbital light modulations in KIC 9406652. We here perform numerical modeling of the observed orbital light curves for a much wider range in the tilt angle with $0.5-4.5 \mathrm{deg}$ as well. As will be shown in subsection 5.2, the best value of the irradiation efficiency, $\eta$, is determined only by the inclination and it is almost independent of the tilt angle. The free parameters to be explored are thus the orbital inclination and the tilt angle.

In what follows, we perform numerical modeling for various orbital inclination and tilt angles. The two different effects produce variations in the orbital light curves, that is, variations in the projected area of the tilted disk and those in the irradiation of the secondary star. The former affects the mean light level of the orbital light curve with different super-orbital phases and the latter affects variations of the orbital light curve profile with different super-orbital phases and these effects are separable. The former effect can be measured by the semi-amplitude
$/ 5$ of the super-orbital light modulations. The latter effect can be (3) measured by the comparison of the observed light curve profiles with the simulated ones. In order to judge which parameters can reproduce the observed light curves the best, we use the following two criteria. The first criterion is the semi-amplitude of super-orbital light modulations, which will be discussed in subsection 5.3. The second one is the goodness of fit between the observed light curves and the model light curves, represented by $\chi^{2}$, the details of which will be explained in subsection 5.6.

We first discuss the efficiency parameter, $\eta$, in subsection 5.2, and we then treat the observational light curve of superorbital light modulations in subsection 5.3. We present a typical example of our modeling for the orbital light curves with eight super-orbital phases, $\varphi_{\mathrm{s}}$, in subsection 5.4 and we discuss the dependence of our results on the tilt angle, $\theta$, and the orbital inclination, $i$, in subsection 5.5. We finally compare simulated light curves for various $i$ and $\theta$ by using the two criteria mentioned above to see which parameter set can reproduce the observed light curves the best.

\subsection{Determination of the irradiation efficiency, $\eta$}

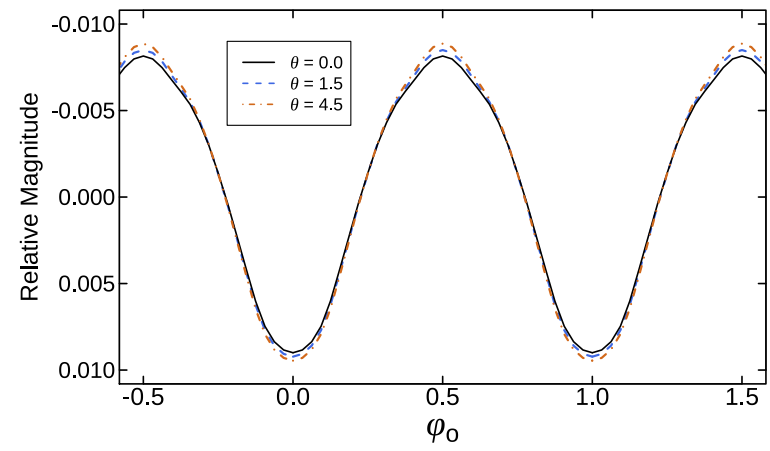

Fig. 4. Averaged orbital signals of the tilted disk of $\theta=1.5$ and 4.5 deg with that of the non-tilted disk. We depict the dashed and dot-dashed lines by averaging the calculated orbital signals of the tilted disk with 8 orientations corresponding to $\varphi_{\mathrm{S}}=0.0,0.125,0.25,0.375,0.5,0.625,0.75$, and 0.875 . The vertical axis is the relative magnitude through the Kepler response function.

The determination of the irradiation efficiency factor is important since the irradiation of the secondary star by the tilted disk plays a major role in our model. In our study, we treat the efficiency factor, $\eta$, to be an adjustable free parameter. We use the orbital light curve of Figure 1 which is obtained by using all data during the brightening stage for this purpose. It has turned out from our modeling that an orbital light curve generated by the superposition of light curves at various orientations of a tilted disk does not depend very much on the tilt angle, $\theta$, and it is basically given by the light curve of a non-tilted disk. Figure 4 displays the averaged orbital signals of the tilted disk of $\theta=1.5$ and $4.5 \mathrm{deg}$ together with that of the non-tilted disk. 
There are only tiny differences between them. Since the orbital light curve of Figure 1 is obtained by all data during the brightening stage, it represents the phase averaged orbital light curve of the tilted disk plus the irradiated secondary star and we can compare Figure 1 with the simulated light curve of the nontilted disk for a given orbital inclination, $i$. We can estimate the best fit value of $\eta$, for various inclinations ranging from 30 to 60 deg with a step of $5 \mathrm{deg}$ and the result is summarized in Table 1. Here we determine the best-fitting parameter, $\eta$, for a given inclination by calculating $\chi^{2}$ between the observed light curve of Figure 1 and model light curves with various $\eta$, with accuracy in 3 figures. In what follows, we use the $\eta$ values given in Table 1 in our modeling, once the orbital inclination is specified.

The best-fitting model light curves for $i=50$ and 60 deg are displayed in Figure 5 together with the observational light curve of Figure 1. As seen in the left panel in Figure 5, the model light curve fits very well with the observational data. On the other hand, the model light curve for $i=60 \mathrm{deg}$ in its right panel deviates from the observational light curve in such a way that a sharp dip due to the eclipse of the outer disk by the secondary is clearly visible around $\varphi_{\mathrm{o}}=0.0$ in the model light curve, while the observed light curve does not show any dip but a rounded minimum. We, therefore, exclude the case of $i=60 \mathrm{deg}$ safely, and hereafter we do not consider this case anymore. We confirm that the best-fitting model light curves for all $i$ except for $60 \mathrm{deg}$ do not show any big differences between the model light curve of $i=50$ deg shown in the left panel of Figure 5 and the others.

\subsection{Light curve of super-orbital modulations and its semi-amplitude}

We construct the light curve of super-orbital modulations during the brightening stage. To do so, two short-period signals (i.e., the orbital and the negative superhump signals) are removed from the original light curve in advance as done in subsection 3.4 of Paper I. We then divide those data into intervals with the length of 4.13 d, i.e., the average period of superorbital signals, perform locally-weighted polynomial regression (LOWESS: Cleveland 1979) to evaluate the intrinsic variation originating from the accretion process for each interval, and subtract it from the light curve. We next fold the light curve by the 4.13-d period and fit the folded one by a sinusoidal curve and set the epoch as the light maximum is $\varphi_{\mathrm{s}}=0.0$. The resulting light curve is shown in Figure 6. We can construct the corresponding model light curve from our modeling. As shown in the next subsection, we construct eight orbital light curves with eight super-orbital phases, $\varphi_{\mathrm{s}}=0.0,0.125,0.25,0.375$, $0.5,0.625,0.75$, and 0.875 for the case of $i=50 \mathrm{deg}$ and $\theta=1.5$ deg corresponding to the right figure of Figure 7. By averaging each light curve, we obtain 8 corresponding fluxes and we show them by blue squares in Figure 6 .
If the super-orbital modulation is produced by the variation in the projected area of the tilted disk with the nodal precession, the expected light curve must be sinusoidal (see, the blue squares in Figure 6). However, the observed light curve shown in Figure 6 deviates from the sinusoidal form. This indicates that the intrinsic variation of the disk luminosity is not properly subtracted from the light curve and some contamination remains. Since the timescale of intrinsic light variations particularly in the brightening stage is of the order of a few days, the contamination to super-orbital modulations from the intrinsic light variation of the accretion disk is inevitable and no good way exists to subtract this effect completely. The intrinsic light variation could lead to either an overestimate or an underestimate in the semi-amplitude of super-orbital modulations. We must take into account this problem in mind. The semiamplitude of normalized super-orbital signals during brightening is $\sim 0.03$ from the orange circles of Figure 6, which corresponds to the tilt angle to be $\sim 1.6 \mathrm{deg}$ for the case of $i=50 \mathrm{deg}$ (see equation (5) in Paper I).

We here discuss another possibility leading to an underestimate in the semi-amplitude of super-orbital modulations. The amplitude of the orbital signal for $\varphi_{\mathrm{s}} \sim 0.5$ is bigger than that for $\varphi_{\mathrm{s}} \sim 0.0$ (see also Figure 2) because the observer looks at the wider high-temperature region of the irradiated surface of the secondary star at $\varphi_{\mathrm{s}} \sim 0.5$, i.e., the irradiation effect seems to be stronger to the observer. The increase in the mean flux level by the irradiation effect is larger at $\varphi_{\mathrm{s}} \sim 0.5$ than that at $\varphi_{\mathrm{s}} \sim 0.0$ because higher-amplitude variations are superimposed at $\varphi_{\mathrm{s}} \sim 0.5$. This causes the semi-amplitude of super-orbital signals to be underestimated. The difference in the normalized semi-amplitude is about 0.0036 between the orbital signal of the top panel of the left column in the right figure of Figure 2 and that of the top panel of the right column in the same figure. The semi-amplitude of super-orbital signals is possibly underestimated by the amount of this difference.

\subsection{Typical example of our modeling for orbital light curves with various super-orbital phases}

Here we describe our simulation method to construct an orbital light curve at a fixed super-orbital phase. We first specify the orbital inclination and the tilt angle of the disk (in this example, $i=50 \mathrm{deg}$ and $\theta=1.5 \mathrm{deg}$ ). We then specify a particular super-orbital phase (for instance, for the case of $\varphi_{\mathrm{s}}=0.125$ in Figure 3) where the zero point of the super-orbital phase is defined when the tilted disk is directly facing the observer. We fix the orientation of the tilted disk in this position but the secondary star orbits around the WD and the tilted disk (counterclockwise as shown in Figure 3). We calculate all fluxes from the tilted accretion disk, the central WD, and the irradiated secondary star at a given orbital phase by the method described in 
Table 1. Relation between the inclination angle $(i)$ in units of degree and the efficiency of irradiation $(\eta)$.

\begin{tabular}{|c|ccccccc|}
\hline$i$ & 30 & 35 & 40 & 45 & 50 & 55 & 60 \\
\hline$\eta$ & 0.577 & 0.477 & 0.399 & 0.337 & 0.286 & 0.241 & 0.191 \\
\hline
\end{tabular}
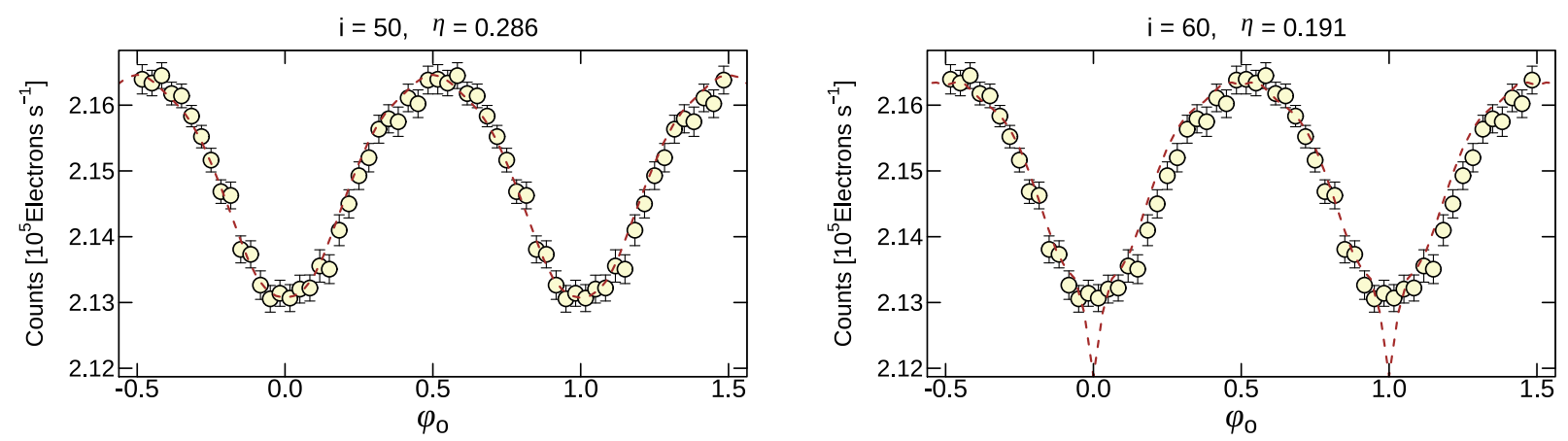

Fig. 5. Model light curves compared with the observational light curve in Figure 1. The dashed line shows the model light curve while the circles with error bar are those of observations.

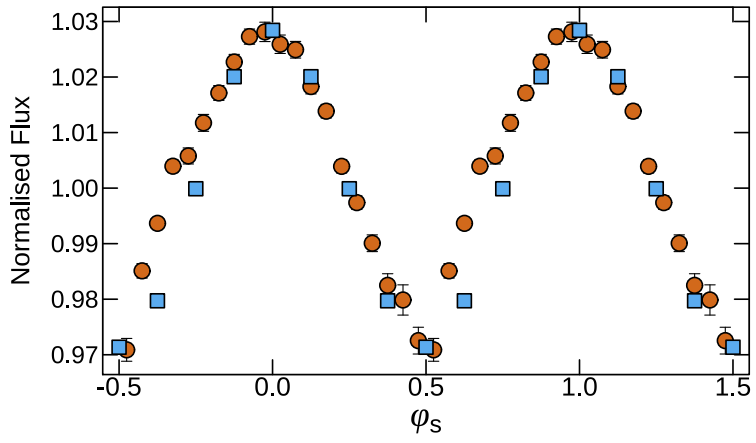

Fig. 6. Phase-averaged profile of super-orbital signals during brightening in KIC 9406652 (the circles) and the prediction from our model light curves of $i$ $=50 \mathrm{deg}$ and $\theta=1.5 \mathrm{deg}$ (the squares). The latter is derived by normalized and averaging each model light curve displayed in the left panel of Figure 7.

section 3. We now construct the orbital light curve for a specified super-orbital phase by calculating fluxes for various orbital phases. We then advance the super-orbital phase by 0.125 by changing the orientation of the tilted disk due to the nodal precession in the retrograde direction (for example, from 0.125 to 0.25 clockwise in Figure 3 ). We finally obtain eight orbital light curves corresponding to eight super-orbital phases and we illustrate our result in the left-hand 8 panels of Figure 7 for the case of $i=50 \mathrm{deg}$ and $\theta=1.5 \mathrm{deg}$.

We find from the eight panels on the left of Figure 7 that the simulated light curves fit fairly well with those observed ones except that the mean light level of the simulation does not agree well with that of observation for some light curves (particularly for the cases of $\varphi_{\mathrm{s}}=0.625$ and 0.75 in the sixth and seventh panels of the left-hand side). As discussed in subsection 5.3, this is mainly caused by our deficiency in subtracting properly the influence of the intrinsic variation from the observed light curves and it is not any deficiency of our modeling. We here compare only the observed and calculated values for the semiamplitude of the super-orbital light signals as our criterion 1 .

As for the second criterion, we rather plot each orbital light curve at a given super-orbital phase by adjusting the mean flux level of the simulated light curve to that of the observational one. The eight orbital light curves in the right-hand side of Figure 7 exhibit our results thus obtained. We see that our simulated light curve profiles reproduce the observed ones fairly well. That is, the most conspicuous observational features are reproduced by our model as the peak of the light curve shifts to earlier orbital phase with the advance of the super-orbital phase and the amplitude of orbital light curve is maximized at the super-orbital phase $\varphi_{\mathrm{s}}=0.5$, and the light minima always occur at $\varphi_{\mathrm{o}}=0.0$.

The simulated orbital light curve profiles are produced by two different effects, that is, the ellipsoidal variation of the secondary star and variations of the irradiated secondary by the tilted disk. These two effects are shown separately in the righthand eight panels of Figure 7. Since the ellipsoidal effect of the secondary star is independent of the orientation of the tilted disk, we show it only in the first panel of the right-hand side figure corresponding to $\varphi_{\mathrm{s}}=0.0$. As well known, two peaks appear at $\varphi_{\mathrm{o}}=0.25$ and 0.75 by the ellipsoidal effect. The amplitude of the simulated orbital light curve at $\varphi_{\mathrm{s}}=0.5$ seems to be smaller than the observed one. It is however difficult to judge by eye how well the simulated light curve profiles reproduce the observed ones and we, therefore, use criterion 2 in subsection 5.6. 

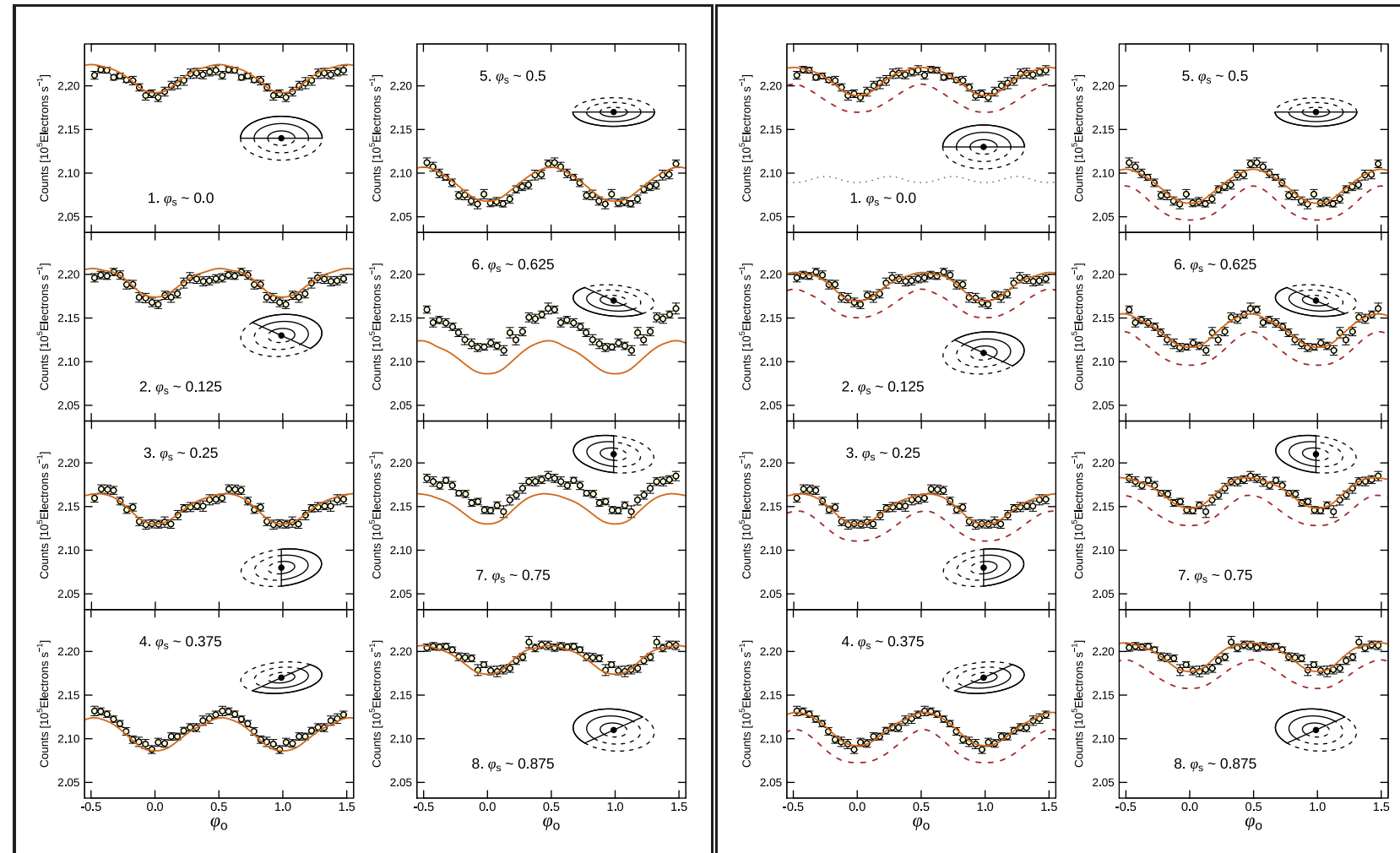

Fig. 7. Comparison of model light curves (red solid lines) with observed ones (dots with error bars) shown in the right figure of Figure 2 for the case of $i=$ $50 \mathrm{deg}$ and $\theta=1.5 \mathrm{deg}$. The eight left-hand side panels illustrate the case when the mean flux level of all model light curves is adjusted to that of the averaged orbital light curve of all super-orbital phases, while the eight right-hand side panels do the case in which the flux level of each model light curve at a given $\varphi_{\mathrm{s}}$ is adjusted to that of the corresponding observed light curve for the purpose of a direct comparison of light curve profile. The model light curve in each panel of the right-hand side is decomposed into two different effects: the irradiation effect and the ellipsoidal modulation of the secondary, and they are shown by dashed and dot lines. The ellipsoidal light curve is displayed only in the top left panel of the right-hand side figures below the irradiation light curve (red dashed curve) because it is the same for all eight $\varphi_{\mathrm{s}}$. The irradiation and ellipsoidal light curves are shifted by $0.06 \times 10^{5}$ and $2.01 \times 10^{5}$ for visibility.

\subsection{Dependence of our modeling on the tilt angle, $\theta$, and the orbital inclination, $i$}

Before evaluating all of the results of our modeling by the two criteria that we define, we first examine the dependence of the results on the tilt angle, $\theta$, by fixing the orbital inclination. Figure 8 illustrates our results for the case with $i=50 \mathrm{deg}$. We see that the amplitude of the orbital light curve for $\varphi_{\mathrm{s}}=0.5$ clearly increases with an increase in the tilt angle while it does not for $\varphi_{\mathrm{s}}=0.0$. In other words, their ratio increases with the increase in $\theta$. We also find that the higher the tilt angle is, the larger the shift of the peak of the orbital light curve is with the advance in the super-orbital phase. We also see that the ellipsoidal effect becomes more important relative to the irradiation effect for $\varphi_{\mathrm{s}}=0.0$, as the tilt angle becomes higher, while the opposite is the case for $\varphi_{\mathrm{s}}=0.5$.

We have also examined the dependence of our results on the orbital inclination for a fixed tilt angle. We find that the ellipsoidal effect of the secondary is most clearly visible for the highest orbital inclination as the two shoulders around the light maximum (for the cases at $\varphi_{\mathrm{s}}=0.0$ and 0.5 ) and that it becomes less clear with a decrease in the orbital inclination. Besides, the shift to an earlier orbital phase of the light maximum with the advance in $\varphi_{\mathrm{s}}$ becomes clearer, the lower the inclination is.

We illustrate the binary configuration of the irradiated secondary star and the tilted disk for four super-orbital phases in Figure 9 to help for understanding our modeling. By comparing the illustration at $\varphi_{\mathrm{s}}=0.0$ (the top panel) and that at $\varphi_{\mathrm{s}}=$ 0.5 (the bottom panel), we see that the irradiated hemisphere of the secondary star is more easily observed at $\varphi_{\mathrm{s}}=0.5$ when the secondary star is at the superior conjunction and therefore the amplitude of the orbital light curve is maximized at this superorbital phase of $\varphi_{\mathrm{s}}=0.5$. The temperature distribution of the irradiated surface is asymmetric with respect to the orbital phase 0.5 at $\varphi_{\mathrm{s}}=0.25$ and 0.75 (see the right and left panels), so that the light maximum of the orbital light curve deviates from the orbital phase 0.5 . The orbital phase at the light maximum thus shifts towards the earlier orbital phase with the advance of the super-orbital phase.

As for the dependence of light curves on the tilt angle of the disk, it is quite natural to understand that the above-mentioned effects are more enhanced if the tilt angle becomes higher. On the other hand, the same is true if the inclination angle becomes 

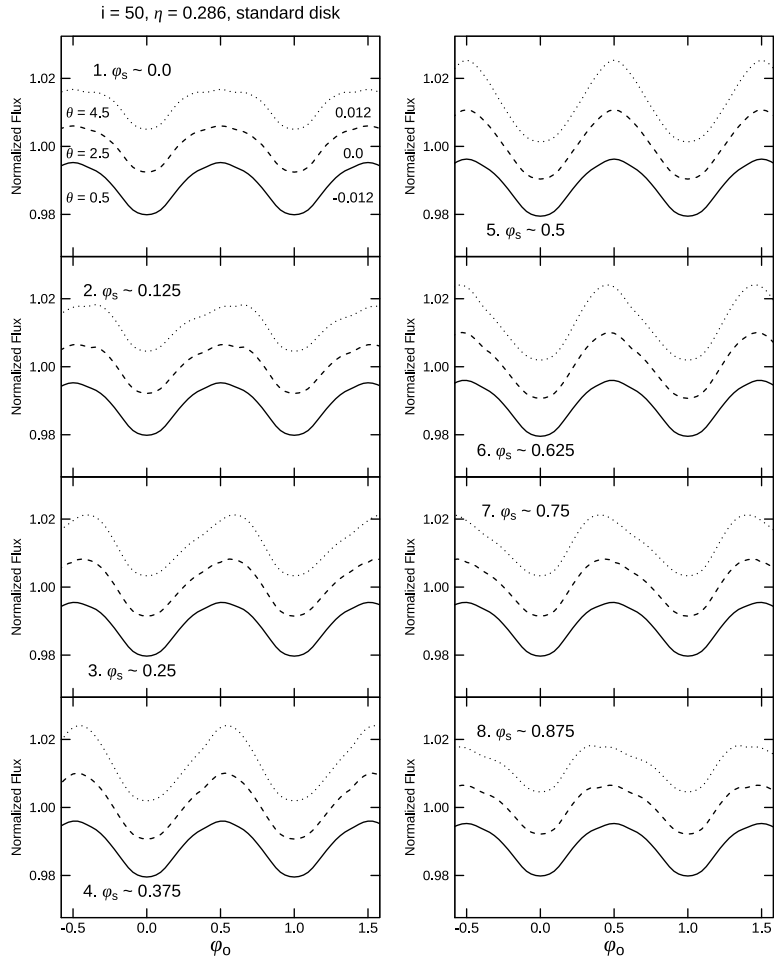

Fig. 8. Dependence of model light curves on the tilt angle for $i=50 \mathrm{deg}$. The tested tilt angles $(\theta)$ are $0.5,2.5$, and $4.5 \mathrm{deg}$ and each resulting orbital profile is shifted in the vertical axis for visibility (see the left top panel).

lower. This is because the northern hemisphere of the secondary star is more easily seen and its southern hemisphere tends to be hidden from the observer if the orbital inclination is lower.

\subsection{Best-fitting parameter set for reproducing the observed orbital light curves}

We have performed orbital light curve simulations for the parameter sets with the orbital inclination $i=30,35,40,45,50$, and $55 \mathrm{deg}$ and with the tilt angle $\theta=0.5,1.5,2.5,3.5$, and 4.5 deg and four more sets for $\theta=2.0 \mathrm{deg}$ with the cases of $i=45$ and $50 \mathrm{deg}$ and for $\theta=3.0 \mathrm{deg}$ with the cases of $i=50$ and $55 \mathrm{deg}$. The results are summarized in Table 2.

As described in subsection 5.1, we use two criteria to judge which parameter set of simulations can best reproduce the observed orbital light curves for various super-orbital phases of the right figure of Figure 2. The first criterion for the semiamplitude of super-orbital light modulations basically measures the variation in the projected area of the tilted disk, and it reflects that the average light levels of individual orbital light curves vary in the super-orbital phase, as shown in the right figure of Figure 2. Its observational value is estimated to be 0.03 from Figure 6.

The second criterion is the goodness of fit between the observed light curves in the right figure of Figure 2 and simulated
Table 2. Summary of the evaluation of our modeling results for the right figure of Figure 2 by the two criteria. Here $\chi_{\nu}^{2}$ stands for reduced $\chi^{2}$.

\begin{tabular}{|c|c|c|c|c|}
\hline$i$ & $\theta$ & Semi-amplitude & $\chi^{2}$ & $\chi_{\nu}^{2}$ \\
\hline 30 & 0.5 & 0.005 & 150 & 0.95 \\
& 1.5 & 0.014 & 132 & 0.84 \\
& 2.5 & 0.023 & 164 & 1.04 \\
& 3.5 & 0.032 & 244 & 1.54 \\
& 4.5 & 0.041 & 375 & 2.38 \\
\hline 35 & 0.5 & 0.006 & 152 & 0.96 \\
& 1.5 & 0.017 & 129 & 0.82 \\
& 2.5 & 0.028 & 143 & 0.91 \\
& 3.5 & 0.039 & 192 & 1.22 \\
& 4.5 & 0.050 & 279 & 1.77 \\
\hline 40 & 0.5 & 0.007 & 154 & 0.98 \\
& 1.5 & 0.020 & 129 & 0.81 \\
& 2.5 & 0.033 & 131 & 0.83 \\
& 3.5 & 0.047 & 160 & 1.01 \\
& 4.5 & 0.060 & 217 & 1.37 \\
\hline 45 & 0.5 & 0.008 & 157 & 0.99 \\
& 1.5 & 0.024 & 131 & 0.83 \\
& 2.0 & 0.032 & 126 & 0.80 \\
& 2.5 & 0.040 & 127 & 0.80 \\
& 3.5 & 0.056 & 143 & 0.90 \\
& 4.5 & 0.072 & 181 & 1.15 \\
\hline 50 & 0.5 & 0.010 & 159 & 1.00 \\
& 1.5 & 0.029 & 133 & 0.84 \\
& 2.0 & 0.038 & 126 & 0.80 \\
& 2.5 & 0.048 & 124 & 0.79 \\
& 3.0 & 0.057 & 125 & 0.79 \\
& 3.5 & 0.067 & 131 & 0.83 \\
& 4.5 & 0.086 & 155 & 0.98 \\
\hline 55 & 0.5 & 0.011 & 161 & 1.02 \\
& 1.5 & 0.034 & 136 & 0.86 \\
& 2.5 & 0.057 & 125 & 0.79 \\
& 3.0 & 0.068 & 124 & 0.78 \\
& 3.5 & 0.080 & 126 & 0.80 \\
& 4.5 & 0.102 & 140 & 0.89 \\
\hline
\end{tabular}

ones, represented by $\chi_{\nu}^{2}$. To calculate this, we first adjust the mean light level of each simulated orbital light curve to that of the observed one in the right figure of Figure 2 in order to examine purely the profile of the orbital light curves. We then calculate $\chi^{2}$ for each orbital light curve by the following equation:

$\chi^{2}=\sum_{i=1}^{N} \frac{\left(F_{i}-F\left(\varphi_{i}\right)\right)^{2}}{\sigma_{i}^{2}}$,

where $N$ is the number of the data per one orbital light curve, and in our case $N=20$, and $F_{i}, F\left(\varphi_{i}\right)$, and $\sigma_{i}$ represent the observed flux, the flux of the model light curve at a specific orbital phase $\left(\varphi_{i}\right)$, and the standard deviation of each observational data point, respectively. We calculate $\chi^{2}$ for one orbital light curve with a given super-orbital phase and sum them up for 


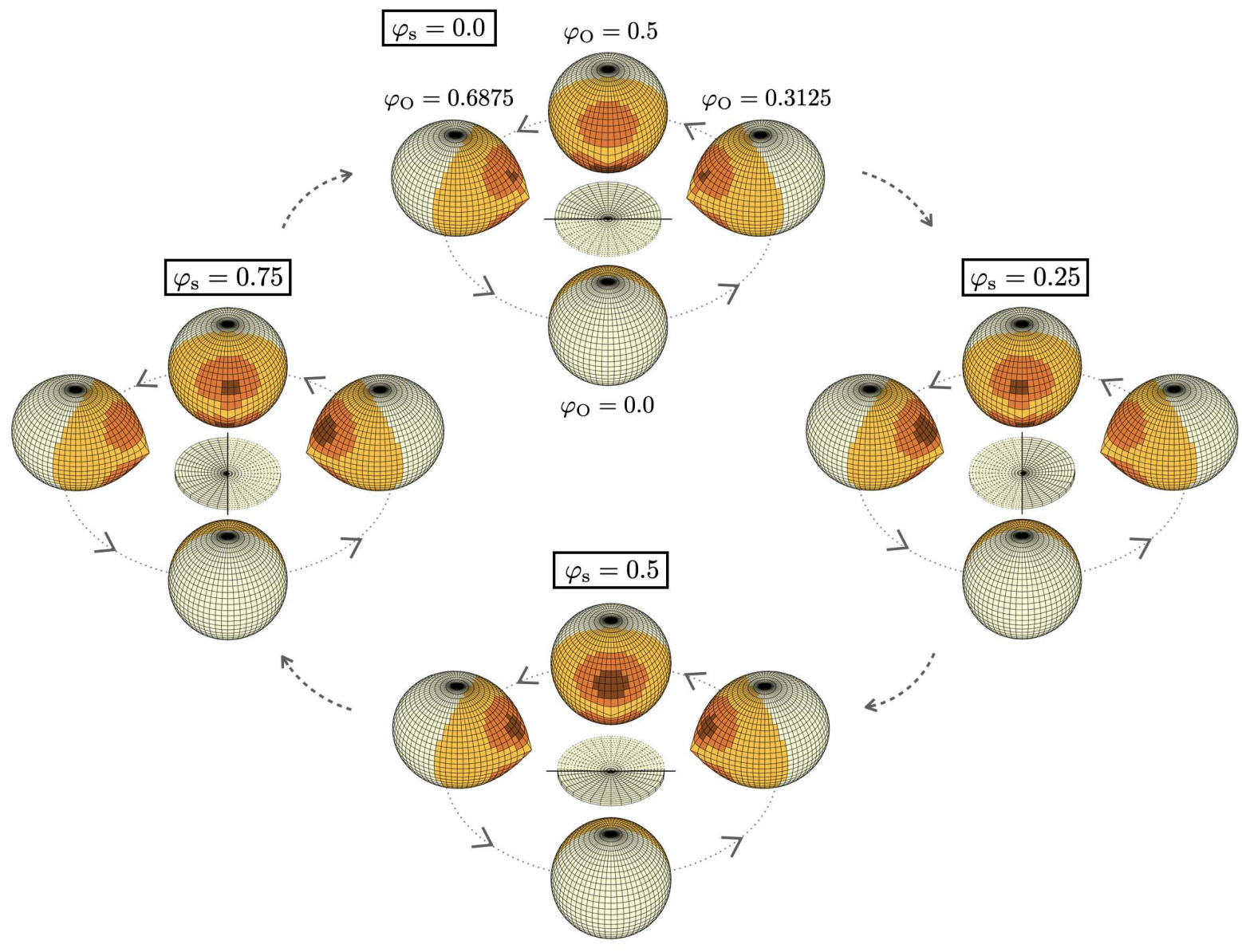

Fig. 9. Binary configurations with four super-orbital phases showing the tilted disk and the irradiated secondary star by colors in the case of $i=50$ deg and $\theta=$ 2.0 deg. The temperature of the irradiated surface of the secondary star is shown in color, i.e., brown for that above $5,000 \mathrm{~K}$, orange for that between $4,500 \mathrm{~K}$ and $5,000 \mathrm{~K}$, and yellow for that below $4,500 \mathrm{~K}$ but above $4,100 \mathrm{~K}$. The super-orbital phase advances in the clockwise direction starting from the top figure for $\varphi_{\mathrm{s}}=0.0$ (where the tilted disk is facing toward the observer) and then $0.25,0.5$, and finally 0.75 . The secondary star makes one orbital revolution around the WD and the tilted disk counterclockwise in each frame, showing the four orbital phases at $\varphi_{0}=0.0,0.3125,0.5$, and 0.6875 . The nodal line of the tilted disk is shown by the diametric line and the solid/dashed parts of the disk represent those for the above/below the orbital plane.

eight orbital light curves. The degree of freedom, $\nu$, is then 158 because the number of total data points is 160 and the number of free parameters in our model is 2 for the orbital inclination and the tilt angle of the disk, respectively. We calculate the reduced $\chi^{2}$ (denoted as $\chi_{\nu}^{2}$ ) by dividing $\chi^{2}$ by $\nu$. The reduced $\chi^{2}$ is also given in Table 2. If it is around unity, the fit is supposed to be good. If it exceeds 1 greatly, the deviation of the model light curve from the observed light curve is too large. On the other hand, if it is extremely less than 1 , it does not mean that the fit is better but it may simply mean that observational errors represented by $\sigma_{i}$ in equation (4) are largely over-estimated. Since our minimum reduced chi-square is around 0.78 and close to 1 , we consider that our modeling is good and that the smaller the chi-square is, the better the fit is.

The results of our evaluation are shown in the $(i, \theta)$ plane of Figure 10. We estimate the best-fitting tilt angle for criterion 1 , which reproduces the observational semi-amplitude of super- orbital signals by linearly interpolating the results in Table 2 for a given orbital inclination (the dots and the line connecting them). As discussed in subsection 5.3, some possible error is inevitable in determining the semi-amplitude of super-orbital signals. We must make some allowance for a possible range of the tilt angle for criterion 1 . If we consider a possible error of $20 \%$ and $30 \%$ for the semi-amplitude with the value of 0.03 , the possible ranges of the tilt angle are the areas surrounded by the dashed and dot-dashed lines in Figure 10.

As for criterion 2, we first set finer grids in the $(i, \theta)$ plane and interpolate $\chi^{2}$ by using the results in Table 2 at each grid point. We interpolate $\chi^{2}$ by a quadratic curve for each tilt angle and do linearly for each inclination angle. We then determine the best-fitting parameter set for $i$ and $\theta$ which minimizes $\chi^{2}$, and display it as the cross in Figure 10. Here the minimum value of $\chi^{2}$ is denoted by $\chi_{\min }^{2}$. If we introduce $\Delta \chi^{2}$ defined as $\chi^{2}-\chi_{\min }^{2}, \Delta \chi^{2}$ follows $\chi^{2}$ distribution with the degree of 


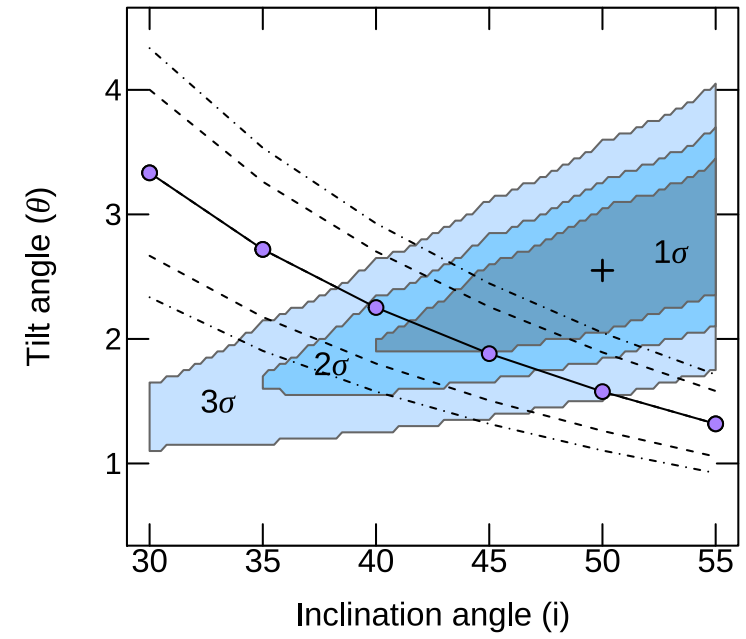

Fig. 10. Best-fitting parameters expected from the results in Table 2. The dots stand for the best-fitting tilt angles for a given orbital inclination based on criterion 1 , which are interpolated from the results of Table 2 . The cross is the best-fitting parameter set for criterion 2 yielding $\chi_{\min }^{2}$. The error regions of $1 \sigma, 2 \sigma$, and $3 \sigma$ of the parameters, which are calculated on the basis of $\Delta \chi^{2}$ test, are drawn by blue contours. If we allow a possible error of $20 \%$ and $30 \%$ for the semi-amplitude of super-orbital signals, the error ranges of the tilt angle for criterion 1 are represented as the areas surrounded by the dashed and dot-dashed lines.

freedom of 2 (Bevington \& Robinson 2003). We plot by blue contours in Figure 10 the distributions of $\Delta \chi^{2} \leq 2.30, \Delta \chi^{2} \leq$ 4.61 , and $\Delta \chi^{2} \leq 9.21$, which correspond to the $1 \sigma, 2 \sigma$, and $3 \sigma$ error regions, respectively.

We see from Figure 10 that the two criteria give opposite dependence with respect to the orbital inclination and we find that the best-fitting parameter set is $i \sim 45 \mathrm{deg}$ and $\theta \sim 2.0 \mathrm{deg}$ to satisfy these two criteria. On the other hand, we have used much more information in evaluating criterion 2 than that by criterion 1. The weights of these two evaluations may not be equal. If we consider only the results of the profile fitting of light curves evaluated by criterion 2 , the best-fitting parameter set is $i \sim 50 \mathrm{deg}$ and $\theta \sim 2.5 \mathrm{deg}$. The acceptable ranges for the inclination angle and the tilt angle of the disk within $1 \sigma$ are around 40-55 deg and 2.0-3.5 deg, respectively. The case of low inclination angle, $\lesssim 35 \mathrm{deg}$, may be ruled out.

\section{Summary and discussion}

\subsection{Summary}

We have first reconstructed observational orbital light curves by subtracting negative superhump signals from the original data, as we did not do this in Paper I. The resulting light curves are found to be greatly modified from the original ones (see also Figure 2). However, the main characteristics remain the same before and after this correction. We have performed the modeling of orbital light curves of KIC 9406652 by treating the retro- gradely precessing tilted disk and the irradiated secondary star in order to reproduce the observed orbital signals in the brightening stage, whose profile varies as the super-orbital phase advances. We have set the inclination angle of the binary system $(i)$ and the tilt angle of the disk $(\theta)$ as free parameters, and carried out simulations with various combinations of these parameter sets, and compared the results with the observational light curves displayed in the right figure of Figure 2. We have found that the observed orbital curves are well reproduced by our modeling (e.g., Figure 7). This confirms our interpretation of Paper I; observed orbital light curves are understood based on our model in which the secondary star is irradiated by the tilted and retrogradely precessing accretion disk, as the secondary star works as a reflecting mirror.

We have explored the best-fitting parameter set of the orbital inclination, $i$, and the tilt angle, $\theta$, of the disk to reproduce the observed light curves. To do so, we have adopted two criteria: (1) the semi-amplitude of the super-orbital signal and (2) the goodness of fit of orbital light curve profiles between observed and calculated ones, represented by the $\chi^{2}$. We have found that the best-fitting parameter set is something around $i \sim 45 \mathrm{deg}$ and $\theta \sim 2.0 \mathrm{deg}$ if we take into account these two criteria equally (see Figure 10). However, the weights of these two criteria may not be necessarily equal as the profile fitting of criterion 2 uses more information than that of the semi-amplitude of criterion 1. If we use criterion 2 only, we have found that the best-fitting parameter set is $i \sim 40-55 \mathrm{deg}$ and $\theta \sim 2.0-3.5 \mathrm{deg}$. The inclination angle lower than $\sim 35$ deg would be ruled out from our results.

In Paper I, we estimated the tilt angle of the disk only by the semi-amplitude of the super-orbital signal, which corresponds to our evaluation by criterion 1 (see the line connecting the dots in Figure 10). However, we figure out from our results that the tilt angle determined solely from this method could be underestimated. The estimation based on the light curve profile fitting gives a systematically higher value of the tilt angle for the orbital inclination of our interest (i.e., $i \sim 45-55 \mathrm{deg}$ ) than that based on the semi-amplitude of the super-orbital signal.

We may add here that we have performed the same modeling for a thicker disk having $H / r \sim 0.1$ at the disk outer edge and compared the results with those for a thinner disk of the standard case. Since the difference between the two is found to be so small, it may not be necessary to show the resulting figure. A tiny difference is that the irradiation effect is slightly larger in the case of the standard disk than that of the thicker one.

We would like to discuss finally an interesting question raised by the referee; are there other negative-superhump stars with bigger amplitudes, which could in principle be used to carry out this kind of analysis? In this respect, KIC 9406652, is unique in the sense that the complete orbital light curves are available for all phases of the super-orbital light modulations 
thanks to observations by the Kepler satellite. Besides, the irradiated component by the tilted accretion disk is clearly visible in its orbital light curves since its accretion disk keeps a bright state in most of the observational time and its inclination angle is neither too low nor too high in this star. Unfortunately, no other similar objects are known with such rich observational data available among CVs, as far as the authors know. On the other hand, there exists at least one star among X-ray binaries in which such rich data are available, which is HZ Her known as the optical counterpart of the X-ray source Her X-1 (Gerend \& Boynton 1976). Her X-1/HZ Her is the famous X-ray binary with the orbital period of $1.7 \mathrm{~d}$ which shows $35-\mathrm{d} \mathrm{X}$-ray ONOFF cycle. This cycle is believed to be produced by occultation of the central X-ray source by the precessing tilted accretion disk. It is known that the optical orbital light curve of HZ Her varies in a highly systematic fashion over the $35-\mathrm{d}$ cycle just in the same way as KIC 9406652. However, the source of irradiation to the secondary star is thought to be the central X-ray source in the case of HZ Her and the precessing tilted disk plays a role as a shadowing object for the irradiated secondary star. In this respect, the situation is different between KIC 9406652 and HZ Her. Also, the orbital inclination of HZ Her is higher than 80 deg (Leahy \& Abdallah 2014). We hope that future observations could provide such rich data for the negative-superhump objects among CVs and the present type of analysis could have a promising return. For example, some of the bright CVs such as V1193 Ori and V751 Cyg listed in Table 5 of Armstrong et al. (2013) have been observed by the Transiting Exoplanet Survey Satellite (TESS) known as the successor of the Kepler satellite, and hence, this kind of analysis would be applicable to these objects once abundant data is collected.

\subsection{Consistency in the estimation of the inclination angle between photometric and spectroscopic approaches}

As discussed in subsection 4.1, the orbital inclination of this system is estimated from the spectroscopic considerations and it is around $i=50 \mathrm{deg}$ (Gies et al. 2013). If uncertainty in the spectroscopic observations is taken into account, its possible range is 45-60 deg. Our estimate of the orbital inclination around 45 deg based solely on the photometric considerations is thus consistent with that from the spectroscopic considerations.

Strictly speaking, the best-fitting inclination angle, $45 \mathrm{deg}$, via our photometric approach is slightly lower than that of 50 deg derived by Gies et al. (2013) via optical spectroscopy. If the difference between these two values matters and if the blame lies on the photometric side, we may here explore possible causes why our modeling yields a lower inclination angle. One possibility is that we were not able to sufficiently subtract the effects of negative superhumps in section 2 . If the effects of negative superhumps had still remained in the observational light curves shown in the right panel of Figure 2, and if we could remove these effects completely, the phase shift of the light maximum and the amplitude variation in orbital light curves would become smaller with the advance of super-orbital phases. The blue contour of Figure 10 would then be shifted towards smaller tilt angles in such a case. As a result, the best-fitting inclination angle would approach towards $50 \mathrm{deg}$. Another possibility is that the semi-amplitude of super-orbital signals could be underestimated. This case would also give a larger inclination angle.

\subsection{Disappearance of the orbital hump during brightening}

In this study, we have treated mainly the irradiated secondary star in the orbital light curve modeling and we have not considered the orbital hump which is supposed to be produced by the collision of the gas stream at the disk rim. In fact, the orbital signal during the brightening stage is dominated by the effect of irradiation of the secondary star and no signal of the orbital hump is visible since the averaged orbital light curve exhibited in Figure 1 peaks at the orbital phase 0.5 and is symmetric with respect to that phase.

On the other hand, we detected the orbital hump peaked at phase $0.8-0.9$ in the orbital signal during quasi-standstills (see the middle panel of Fig. 8 in Paper I). If the luminosity of the bright spot and that of the disk outer edge are the same as those in quasi-standstills, we see an orbital hump with the same amplitude in the flux scale during brightening as that in quasi-standstills. However, the orbital hump seems to have completely disappeared when the system brightness increases only by $\sim 1.6$ times. That looks rather strange.

In ordinary U Gem-type stars, the orbital hump (which is a dominant feature in the orbital light curve in quiescence) disappears during outburst, because the flux ratio of the bright spot with respect to the disk outer edge drastically increases in comparison with that in quiescence. One possibility is that the outer part of the disk at quasi-standstills in KIC 9406652 could be in the cool state so that the bight spot is conspicuous. In fact, we proposed the model for the light variation of IW And-type stars including this object in Kimura et al. (2020a), in which only the outer part of the disk is in the cool state during quasi-standstills. However, this was not supported by the observed frequency variation of negative superhumps, which suggests that the disk gradually expands during quasi-standstills (see Paper I). If the outer disk is cool, the disk gradually shrinks due to the inflow of material having small angular momentum to the disk outer edge. The observation of negative superhumps rather implies that the outer disk is in the hot state during quasi-standstills. We have no good idea for explaining the disappearance of or- 
bital humps during brightening and it may remain one of the future problems.

\section{Acknowledgements}

M. Kimura acknowledges support by the Special Postdoctoral Researchers Program at RIKEN. The original numerical code that we revised in this paper was provided by Izumi Hachisu. This work was financially supported by Japan Society for the Promotion of Science Grants-in-Aid for Scientific Research (KAKENHI) Grant Number JP20K22374 (MK). We thank the anonymous referee for helpful comments.

\section{References}

Armstrong, E., et al. 2013, MNRAS, 435, 707

Bailer-Jones, C. A. L., Rybizki, J., Fouesneau, M., Mantelet, G., \& Andrae, R. 2018, AJ, 156, 58

Bevington, P. R., \& Robinson, K. D. 2003, Data reduction and error analysis for the physical sciences; 3rd ed. (McGraw-Hill). New York, NY

Bonnet-Bidaud, J. M., Motch, C., \& Mouchet, M. 1985, A\&A, 143, 313

Cleveland, W. S. 1979, J. Amer. Statist. Assoc., 74, 829

Davis, A. B., Shappee, B. J., Archer Shappee, B., \& ASAS-SN 2015, American Astron. Soc. Meeting Abstracts, 225, \#344.02

Gerend, D., \& Boynton, P. E. 1976, ApJ, 209, 562

Gies, D. R., et al. 2013, ApJ, 775, 64

Godon, P., Shara, M. M., Sion, E. M., \& Zurek, D. 2017, ApJ, 850, 146

Hachisu, I., \& Kato, M. 2001, ApJ, 558, 323

Hameury, J.-M., Menou, K., Dubus, G., Lasota, J.-P., \& Hure, J.-M. 1998, MNRAS, 298, 1048

Harvey, D., Skillman, D. R., Patterson, J., \& Ringwald, F. A. 1995, PASP, 107,551

Kato, T. 2019, PASJ, 71, 20

Katz, J. I. 1973, Nature Physical Science, 246, 87

Kimura, M., Osaki, Y., Kato, T., \& Mineshige, S. 2020a, PASJ, 72, 22

Kimura, M., Osaki, Y., \& Kato, T. 2020b, PASJ, 72, 94 (Paper I)

Knigge, C. 2006, MNRAS, 373, 484

Leahy, D. A., \& Abdallah, M. H. 2014, ApJ, 793, 79

Leibowitz, E. M. 1984, MNRAS, 210, 279

Lubow, S. H. 1992, ApJ, 398, 525

Mann, Andrew W., \& von Braun, Kaspar 2015, PASP, 127, 102

Montgomery, M. M. 2012, ApJ, 745, L25

Montgomery, M. M., \& Martin, E. L. 2010, ApJ, 722, 989

Motch, C. 1981, A\&A, 100, 277

Murray, J. R., Chakrabarty, D., Wynn, G. A., \& Kramer, L. 2002, MNRAS, 335, 247

Paczynski, B. 1977, ApJ, 216, 822

Patterson, J. 1999, in Disk Instabilities in Close Binary Systems, ed. S. Mineshige, \& J. C. Wheeler (Tokyo: Universal Academy Press), p. 61

Patterson, J., et al. 2002, PASP, 114, 1364

Patterson, J., Kemp, J., Saad, J., Skillman, D. R., Harvey, D., Fried, R., Thorstensen, J. R., \& Ashley, R. 1997, PASP, 109, 468

Shakura, N. I., \& Sunyaev, R. A. 1973, A\&A, 24, 337

Smak, J. 1992, Acta Astron., 42, 323
Smak, J. 2009, Acta Astron., 59, 419

Warner, B. 1995, Cataclysmic Variable Stars (Cambridge: Cambridge University Press)

Wood, M. A., \& Burke, C. J. 2007, ApJ, 661, 1042

Wood, M. A., Montgomery, M. M., \& Simpson, J. C. 2000, ApJ, 535, L39 